\title{
Gestión Gerencial en el Sector Agropecuario TRADICIONAL COLOMBIANO*
}

\author{
Ignacio Aguilar Zuluaga*** \\ Luis Ángel Madrid****
}

Semillero de investigación (Escuela de Negocios):

Carlos Archila, Cristina Castrillón, Jorge Combariza, Gonzalo Sánchez.

\section{Resumen}

Nuestra investigación, de la cual este escrito es un avance, indaga acerca del estado del manejo o administración de fincas, granjas y parcelas rurales, localizadas en la Altiplanicie cundiboyacense, dedicadas a cultivos y ganaderías propias de la región.

Se parte de la hipótesis de que esas fincas rurales tienen una administración muy deficiente o nula, llevada a cabo en forma empírica y tradicional, lo cual las margina de los métodos y sistemas propios de una gestión gerencial eficiente y moderna, todo lo cual contribuye a bajos índices de productividad.

El proyecto busca resolver la hipótesis a través de sus objetivos, que son: destacar la importancia económicosocial del sector, conocer su situación económicosocial actual y las maneras como se manejan sus unidades de producción. Además, apunta a determinar los incentivos, las políticas y las estrategias necesarias para administrar las empresas de sector agropecuario tradicional.

\begin{abstract}
This paper surveys managerial practices of small farms and land plots in the Cundinamarca and Boyacá highlands. The hypothesis advanced is that in these agricultural units, business practices are nearly non-existent or marginal. Conversely, management is carried out in traditional and empirical ways, devoid of modern methods.

The project aims to solve the hypothesis through its own stated objectives: examining the socio-economic situation and importance of small Colombian agriculture in the area together with the current managerial practices. Finally, it is geared to suggest public policies, strategies and incentives to further traditional Colombian agribusiness.
\end{abstract}

\section{Palabras clave}

Agropecuaria, sector tradicional, gestión gerencial, eficiencia, productividad, fincas rurales, capacitación, campesino.

\footnotetext{
* Este artículo es un avance de la investigación que los autores adelantan sobre la situación gerencial de los pequeños productores del altiplano cundiboyacense, inscrita en el Comité de Investigaciones de la Escuela de Empresa de la Universidad Sergio Arboleda. Recibido el 10 diciembre 2008, aprobado, el 10 de junio de 2009

** Economista de la Universidad de los Andes, especializado en Administración Industrial y Alta Gerencia; Diplomado en Gerencia Internacional Autor de varios libros, ensayos y artículos sobre temas económicos. Docente investigador de la Escuela de Empresa de la Universidad Sergic Arboleda. Correo electrónico; ignacio.aguilar@usa.edu.co.

**** Abogado de la Universidad del Rosario, LLM del London School of Economics, LLM en International Economic Law de la Universidad de Warwick (UK) y Especialista en Negociaciones y Relaciones Internacionales de la Universidad de los Andes. Docente investigador de la Escuela de Empresa de la Universidad Sergio Arboleda. Correo electrónico: luis.madrid@usa.edu.co.
} 


\section{Keywords}

Agro-business; peasant sector, business management, efficiency, productivity, rural farms, training, small farmers.

JEL: N56; O 13; O।8; Q I2; Q I5

\section{Introducción}

\section{Objetivos}

Principal

Investigar la gestión gerencial en las fincas rurales de la Altiplanicie cundiboyacense, para determinar los métodos y prácticas administrativos que sus propietarios y administradores llevan a cabo para su explotación y desarrollo.

\section{Específicos}

- Investigar las condiciones y situación de la producción y la productividad del sector agropecuario tradicional de la altiplanicie cundiboyacense.

- Examinar los métodos de administración de las fincas localizadas en la región escogida como muestra representativa.

- Determinar la participación de la gestión gerencial de las fincas agropecuarias de la región seleccionada, en sus resultados económicos y financieros.

\section{Marco de Referencia}

\section{Teórico}

La investigación está enmarcada dentro de las teorías y postulados que se refieren y se relacionan con los sistemas de administración agropecuaria y con el empleo de los recursos de producción para obtener resultados positivos y aceptables de la inversión en el sector agropecuario nacional, medidos como tasas de rentabilidad comparadas (y comparables) con el sector urbano.
Incluye las teorías sobre las políticas macro y microeconómicas que deben aplicarse en la realización de una gestión gerencial eficiente de las fincas agropecuarias y, además, identifica las políticas y estrategias gubernamentales dictadas para impulsar y promover el desarrollo sostenible y creciente del sector agropecuario tradicional.

\section{Conceptual}

La investigación se enmarca dentro de los conceptos, principios y teorías conocidos y aceptados universalmente, pertenecientes a economistas, académicos y tratadistas, quienes se han ocupado del estudio y análisis rigurosos (así como de sus aplicaciones), de teorías estrategias y políticas relacionados con la conformación, la importancia y el papel que tiene el sector agropecuario de un país en proceso de su desarrollo económico y social.

\section{Espacial}

La investigación se limitó al estudio y examen de las condiciones de las fincas seleccionadas en treinta y seis municipios de la Altiplanicie cundiboyacense de Colombia.

\section{Hipótesis}

\section{Principal}

Con una gestión gerencial eficaz, eficiente y moderna, las fincas, haciendas o granjas agropecuarias, pertenecientes al sector tradicional o campesino, elevarán sus índices de producción, de productividad y rentabilidad, para contribuir así a aumentar la eficiencia de este sector de la economía colombiana.

\section{Secundaria}

Entre las alternativas existentes para promover el crecimiento y reducir la pobreza y la desigualdad de la población campesina, los recursos y políticas que se orienten y se apliquen a la educación, formación y capacitación de la fuerza laboral que trabaja en el campo se identifican como las más efectivas para el alcance de esos objetivos. 


\section{Metodología}

Se llevó a cabo un trabajo de campo consistente en la realización de 386 encuestas al mismo número de fincas, localizadas en 36 municipios de la Altiplanicie cundiboyacense. Por lo tanto, excluye todas aquellas unidades de producción agroindustrial, cuya administración se lleva a cabo con un criterio y unos métodos empresariales.

El formulario para la encuesta contiene cuarenta y dos (42) preguntas dirigidas a las personas dedicadas a las faenas correspondientes a cada una de las fincas rurales En este documento se analizan los resultados clasificados y tabulados de las respuestas, para apoyar y comprobar los supuestos y la hipótesis planteados para la investigación. Se anexa el formulario empleado (Anexo No. 2).

Se hizo una búsqueda intensa y un análisis detenido de las publicaciones nacionales y extranjeras, referentes a los temas sobre los cuales trata la investigación y se consultaron libros, informes, documentos y ensayos recientes que permiten reseñar y determinar diferentes conocimientos acerca de las causas, evolución y condiciones del sector agropecuario tradicional de la región, así como de la situación que vive su población respecto a niveles de ingresos, desigualdad y pobreza.

Lo anterior se complementa con entrevistas personales y reuniones con altos funcionarios de entidades públicas y privadas que conforman un núcleo amplio de fuentes primarias de información. Esta tarea se hizo principalmente en Bogotá y en algunos municipios del Altiplano (Chía, Sopó, La Calera, Tabio y Tenjo, por ejemplo).

El análisis de las series estadísticas correspondientes a los diez últimos años ha sido utilizado para precisar apreciaciones y confirmar supuestos que permiten determinar la validez de la hipótesis.

\section{Selección de la muestra}

Sudman (1976) indica que el tamaño de la muestra estadística depende de cuatro factores: I. El número de grupos y subgrupos en la muestra que se va a analizar. Para el presente estudio se partió de la hipótesis de la existencia de un solo grupo en esa muestra, la cual corresponde a las fincas del Altiplano cundiboyacense. 2. El segundo factor: la exactitud en los resultados. Como la investigación no pretendía ser el 100\% concluyente sino tener un punto de partida para plantear hipótesis para nuevas investigaciones, consideramos aceptable un nivel de precisión del 5\% (es decir, un nivel de confianza del 95\%). 3. El tercer factor es la relación costo/beneficio del estudio: es aceptable justificar un costo de una muestra más grande, si los costos de muestreo son bajos, pero dada la limitación de tiempo y el presupuesto del proyecto de investigación, consideramos un punto intermedio en términos del tamaño. 4. El cuarto factor se refiere al grado de variación en la población objetivo, la cual para el presente estudio no presenta gran dinamismo en virtud al relativamente corto período de análisis. Por eso consideramos una muestra de 386 encuestas en igual número de grupos (fincas rurales), como representativa y válida desde el punto de vista estadístico.

\section{El Problema}

El sector agropecuario tradicional tiene unas condiciones de explotación, operatividad y productividad, que se suponen identificadas con un alto índice de ineficiencia y pobres resultados en cuanto a volúmenes de producción, costos y calidad. Esto significa una barrera para el crecimiento del sector y para el desarrollo económico del país, representada en desempleo, bajos ingresos y un bajo nivel de vida para la población campesina. Su aporte al PIB, a la generación de empleo y sus cambios en la conformación de la producción, manifiestan ineficiencia en la agropecuaria colombiana (véanse los cuadros 1 y 2).

Se cree que una de las causas de esta situación es su insuficiente, o ausente, administración en la mayoría de las unidades de producción, lo cual puede conducir a una producción deficiente de los renglones cultivados y de la ganadería que se explota.

¿Una administración eficiente, con un criterio verdaderamente empresarial, 
contribuiría a la solución de estos problemas y a lograr que este sector sea más productivo y contribuya así al aumento de su aporte al PIB?

Se supone que la respuesta a esta pregunta debe ser afirmativa, toda vez que es posible establecer, por experiencias y comportamientos actuales, que cuando este tipo de empresas se administran con profesionalismo y eficiencia, sus resultados en términos de productividad, producción y rentabilidad de la inversión, son sensiblemente más altos. Los resultados de la investigación van a servir para comprobar o modificar esta apreciación.

No se puede desconocer que existe una brecha muy grande entre los criterios y las políticas de administración y gerencia de las empresas agroindustriales (las dedicadas a la producción de banano, cacao, flores, palma africana, como ejemplos) y las "empresas" que producen los renglones agropecuarios tradicionales, la mayoría de los cuales se refiere a productos de pancoger (maíz, fríjol, papa, yuca, plátano hartón, hortalizas, frutas).

Para enfatizar el supuesto de la afirmación anterior, puede hacerse la siguiente comparación: cuando una persona, individual y directamente, funda y organiza un establecimiento comercial o industrial en un centro urbano, crea una infraestructura administrativa que comprende gerencia, ventas, contabilidad, personal y servicios generales. El administrador o gerente se selecciona de acuerdo con su preparación académica y su experiencia. Lo mismo se aplica a los cargos para los diferentes departamentos o secciones de la empresa. $Y$ esto es válido aun para microempresas, constituidas con inversiones relativamente bajas.

En cambio, una finca o una hacienda rural, en la cual la inversión es igual o mucho más valiosa, tiene como administrador o "gerente" (llamado mayordomo o "encargado") a un campesino de baja escolaridad, y carece de la infraestructura administrativa necesaria para llevar a cabo una gestión con criterio empresarial.
Por otra parte, es necesario considerar la actitud de muchos propietarios de fincas rurales, la cual se traduce sólo en la expectativa de una valorización de la tierra, o lo que se conoce como "incremento no ganado". A este grupo o tipo de "hacendados", agricultores o ganaderos, no les interesa el rendimiento y la productividad de su finca, porque se atienen a la valorización constante que adquiere la tierra; un activo cuya oferta es inelástica y cuya demanda es elástica, representada en una curva ascendente o positiva. Y, por lo tanto, no la administran como una verdadera empresa.

Por otra parte, el sistema de tenencia y propiedad de la tierra rural contribuye a enfatizar la ausencia o limitación de su administración con criterio y métodos empresariales. En Colombia el estatuto tributario continúa siendo poco efectivo para lograr que las propiedades rurales se exploten adecuada y permanentemente, con el fin de que su rentabilidad permita, a su vez, convertirla en sujeto de tributación.

Debe recordarse que Colombia tiene 42 millones de hectáreas disponibles y aptas para la ganadería y de éstas únicamente se aprovechan 22 millones. Dispone de 13 millones de hectáreas aptas para ser cultivadas y sólo se utilizan 4.6 millones.

El tamaño promedio de las fincas y parcelas campestres se identifica con la presencia de miles de minifundios que tienen un área promedio de seis hectáreas y con la existencia de menos de 1.500 fincas rurales con una extensión de mil hectáreas. Es decir, el sector agropecuario tradicional es en Colombia de tipo minifundista y no latifundista; representado por miles de predios cuya explotación, por su área individual promedio, no es económicamente explotable ni rentable. Este es otro factor más que presenta la carencia del criterio de administración empresarial en este sector de la estructura económica nacional. Se exceptúan las fincas ganaderas que se explotan extensivamente en áreas de mayor dimensión, ubicadas en zonas distintas al objeto de esta investigación, situadas en zonas que no son materia de este estudio. 


\section{La productividad del sector}

La producción y la productividad del sector agropecuario dependen de varios factores. En primer término, de la calidad de la tierra, basada en su composición, en su fertilidad y en el aprovechamiento o suministro del agua. En segundo lugar, de los sistemas empleados en su explotación: extensiva, intensiva, con empleo de maquinaria o sólo de mano de obra; empleo de agroquímicos y fertilizantes. Un tercer factor se refiere al uso de semillas seleccionadas y mejoradas (si se trata de cultivos), y si se trata de ganados de cría, levante o ceba, emplear en su explotación ejemplares puros o cruzados, y si ellos se cuidan o se protegen contra las enfermedades o epizootias.

La capacitación, formación y experiencia de la mano de obra, que trabaja en la finca, constituye otro factor adicional que incide directamente en los resultados de la explotación del predio o parcela en cuestión.

El tamaño o extensión superficiaria de la finca también influye en su producción y en su productividad: los minifundios, que son la mayoría de las "empresas" agropecuarias de la Altiplanicie cundiboyacense, por su extensión reducida a un promedio de tres hectáreas, se manifiestan como unidades no económicamente explotadas y, por consiguiente, con un índice de rentabilidad muy bajo o inexistente.

A todo lo anotado anteriormente, es necesario adicionarle el factor administrativo o gerencial. De acuerdo con la investigación realizada, este es un aspecto que genera preocupaciones y, por consiguiente, se deben proponer estrategias y métodos que lleven a la solución adecuada del problema. Precisamente este es el objetivo principal del presente estudio: dar a la luz pública las respuestas correspondientes a la hipótesis planteada y a los supuestos anotados.

Para llegar a precisar el grado de administración o gerencia que tiene la agropecuaria campesina, en la Altiplanicie cundiboyacense, es preciso considerar los factores relacionados con la preparación y la formación de sus administradores y de todo su personal operativo.

\section{Justificación}

Si se examinan con atención los conceptos y aspectos mencionados en los apartes relacionados con el planteamiento del problema, se comprende la justificación y la importancia de realizar esta investigación.

No obstante, es necesario plantear en forma más específica algunos temas que permitan la demostración, no solamente teórica, de la justificación y pertinencia del estudio al cual pertenece el presente avance.

Las limitaciones administrativas y de gestión empresarial en el sector agropecuario tradicional pueden conducir a un desempeño y a unos resultados deficientes, representados en un desperdicio de los recursos, un bajo aporte del sector al valor del PIB y unos índices de pobreza y marginalidad de la población campesina. Véase el cuadro No. 3.

El que Colombia, país que tradicionalmente ha sido considerado con "vocación agrícola", posea un sector agropecuario tradicional que adolece de las deficiencias ya expuestas, justifica y requiere realizar una investigación que demuestre la situación real del desempeño y los resultados de las unidades de producción que lo conforman.

Si la problemática de este sector se orienta hacia las condiciones actuales que presentan indicadores como el desempleo y el subempleo, la remuneración de la mano de obra, las condiciones de pobreza y el bajo nivel de vida de la mayoría de las familias campesinas, suponemos que este proyecto de investigación es necesario e importante, tanto desde el punto de vista académico, como desde sus aspectos materiales y prácticos (económicos y sociales).

Se espera que la investigación plantee y genere los métodos, las estrategias y las políticas que deben emplearse para que el sector agropecuario tradicional salga de su estado actual y contribuya amplia y positivamente al proceso del desarrollo económico y social del país. Si esto se logra, la realización de este estudio se habrá justificado plenamente (ver cuadros Nos. 4 y 5). 
Con el fin de enfatizar la justificación de la investigación, es pertinente anotar algunos datos e indicadores que respaldan los supuestos relacionados con el estado actual del sector (DNP 2005).

\begin{tabular}{|c|c|}
\hline $\begin{array}{l}\text { - Porcentaje de población que vive en el } \\
\text { campo: }\end{array}$ & 23\% (aprox. 10 millones de personas) \\
\hline $\begin{array}{l}\text { - Remuneración promedio del trabajador } \\
\text { campesino: }\end{array}$ & $65 \%$ del SMML \\
\hline - Población bajo el nivel de la pobreza: & $67 \%$ \\
\hline $\begin{array}{l}\text { - Población en condiciones de extrema } \\
\text { pobreza: }\end{array}$ & $22 \%$ \\
\hline - Déficit de vivienda rural: & I.200.000 unidades \\
\hline - Cubrimiento de la educación primaria: & $72 \%$ \\
\hline $\begin{array}{l}\text { - Cubrimiento de los servicios públicos } \\
\text { esenciales: }\end{array}$ & $68 \%$ \\
\hline - Cubrimiento de salud y seguridad social: & $62 \%$ \\
\hline
\end{tabular}

Al parecer, es evidente que la presencia de esos datos es consecuencia de las debilidades y las fallas que actualmente se observan en la gestión de la agropecuaria tradicional. Una gestión empresarial eficiente, técnica y moderna sugeriría unas condiciones económico sociales menos precarias y un aporte del sector agropecuario tradicional de mayor valor agregado a la economía nacional.

Un argumento adicional que justifica la realización de este proyecto de investigación, se refiere a la especificación de la importancia del sector agropecuario, la cual se determina por lo siguiente (Aguilar Z, 2008):

I. Su contribución al Producto Interno Bruto (PIB).

2. El número de personas que emplea.

3. El ingreso anual por habitante en las zonas rurales.

4. Su alto índice de pobreza.

5. Su aporte al sector externo por medio de las exportaciones de productos agropecuarios.

Por otra parte, debe considerarse que la necesidad de crear incentivos, idear estrategias y establecer políticas, todo ello dirigido a la creación y organización de empresas en el sector agropecuario tradicional, reafirma y enfatiza la justificación de una investigación como la que se desarrolla en este escrito.

Se debe ratificar que la zona escogida como muestra representativa del sector agrícola tradicional exige la presencia de unidades de producción (fincas, granjas, haciendas o parcelas), que se administren con el criterio empresarial con el cual se manejan las agroindustrias y las empresas urbanas, en su gran mayoría.

Lo anterior conduce a aceptar como una necesidad imperiosa, la formulación de un modelo de gestión empresarial que hoy no existe en las fincas campesinas. El modelo (que va a ser considerado como un resultado de la investigación), debe considerarse también como un instrumento de capacitación y formación en administración agropecuaria para el fortalecimiento y la modernización del sector.

Naturalmente, el modelo de administración eficiente de una finca rural tiene que incluir todas las tareas y esfuerzos necesarios para capacitar y entrenar, tanto a quienes van a ser los administradores o gerentes, como a toda la planta de personal que la finca requiere (véase anexo No. 3). 
Llevar al campo el criterio de gestión empresarial, implica la conformación y puesta en práctica de varios aspectos: uno de ellos se refiere a los incentivos que es necesario ofrecer a propietarios de fincas; las estrategias y las políticas que conduzcan a que los propietarios de predios rurales inexplorados o mal administrados, se obliguen a utilizarlos y a administrarlos eficiente y permanentemente.

Si esas políticas e incentivos que motivan la explotación y desarrollo del sector agropecuario campesino o tradicional, se traducen también en una rentabilidad normal promedio, la inversión en el campo se vuelve atractiva.

En otros términos: es preciso que las actividades agropecuarias tradicionales sean un buen negocio, una inversión comparable con las que se hacen en otros sectores de la economía.

Es previsible y justificable, también. que el Estado se comprometa y se interese más por este sector. Es decir, que ejerza una mayor presencia y permanencia.

Todo lo anterior ratifica la necesidad de investigar su estado actual por medio de un estudio como el que se presenta en este documento.

\section{Resultados}

Siguiendo el orden de las preguntas contenidas en el cuestionario de la encuesta, su clasificación y tabulación arrojaron los siguientes resultados:

I. Extensión superficiaria total de las fincas encuestadas: 1.775,6 hectáreas. De este total corresponden a fanegadas el $89 \%$ y un $11 \%$ a hectáreas. El área promedio en fanegadas es de 6,06 y de 4,60 hectáreas por finca. ${ }^{1}$

Se comprueba la existencia de un sistema de tenencia y propiedad de la tierra claramente minifundista. Ello conduce a observar que las fincas investigadas tienen, por su baja extensión, un factor tradicional que contribuye al aumento de los costos de su explotación y a un bajo índice de productividad y de producción.

2. En cuanto al sistema de tenencia de la tierra, el $67 \%$ de las fincas es de propiedad privada (sus dueños las explotan y manejan directamente); un 31\% de las fincas las trabajan campesinos arrendatarios y un 2\% tiene contratos de aparcería.

3. El 44\% de las fincas está dedicado a la ganadería, así: un 40.5\% está dedicado a la ganadería vacuna y se especializa en la producción de leche el 98.9\%. En ganadería ovina se ocupa el $1.5 \%$ y en ganadería porcina también el 1.5\%. Sólo un $1 \%$ de las fincas se ocupa en otros tipos de ganadería (avícola y caballar).

4. La explotación agrícola la lleva a cabo el $56 \%$ de las fincas encuestadas y los cultivos principales son, en su orden, papa (19.5\%), maíz (5.5\%), arveja (4.5\%), hortalizas (3.5\%), lechuga (3.4\%) y curuba (.19\%). El 16.7\% de las fincas está dedicado al cultivo de 33 productos agrícolas más, cada uno de ellos con una participación inferior al 1.2\%. Entre esos cultivos minoritarios se destacan el cilantro, las fresas, el fríjol, la papa criolla y la zanahoria.

5. En lo que se refiere al factor de producción mano de obra o fuerza laboral, la investigación arrojó que un 55.3\% del personal se dedica a labores directas de producción y un $14.7 \%$ a las actividades administrativas. Del total del personal vinculado a las fincas, un 38\% no tiene funciones o labores definidas y permanentes. Con un contrato de trabaio permanente labora el 70\% del personal y un 30\% son temporales; es decir, para tiempos de cosecha o para actividades esporádicas u ocasionales.

6. Respecto al nivel de escolaridad el resultado fue el siguiente:

${ }^{1}$ La fanegada prima en la zona de Cundinamarca y la hectárea en la zona del departamento de Boyacá 
Sin ninguna escolaridad $4.6 \%$

(analfabetismo absoluto):

Con estudios básicos primarios .......54.0\%

(los 5 primeros grados):

Con estudios secundarios ............31.1\%

o bachillerato:

Personal con formación ................. 3.9\%

a nivel técnico:

Personal con estudios

$6.4 \%$

universitarios:

Esos porcentajes están indicando una de las causas más sobresalientes que permite confirmar la pobre o deficiente gestión gerencial, eficiente y moderna, en la región rural dedicada a actividades agropecuarias y tomada como muestra representativa del sector campesino o tradicional (Altiplanicie cundiboyacense).

Adicionalmente a la información anterior, el trabajo de campo indicó que el 61.7\% de las personas que laboran en las fincas encuestadas, corresponde a miembros de la familia propietaria y administradora de la finca.

7. En cuanto se refiere al factor o recurso de producción representado en maquinaria y equipos, se encontró que un $61.7 \%$ emplea maquinaria y equipos agrícolas y el $38.3 \%$ de las fincas de la región no utiliza ningún tipo de máquinas. Así mismo, del porcentaje que usa maquinaria y equipos, un $72,4 \%$ es propietario de estos y el $27.5 \%$ los tiene y emplea como un préstamo de sus vecinos y colegas.

A la pregunta correspondiente a la clase o tipo de equipos, se observa la pre-sencia de fumigadoras (26.9\%), tractores (26.5\%), motobombas (15.1\%), equipos para ordeño (5.2\%) y equipos para riego (4.5\%).

8. Las encuestas realizadas en las 386 fincas revelaron que el $71.1 \%$ emplea agroquímicos y el $28.9 \%$ no los utiliza.

Así, tanto por la gran proporción de fincas que usa maquinarias y equipos, como la que emplea agroquímicos, estarían indicando algún grado de actualización, modernidad o tecnificación del sector agropecuario tradicional de la región escogida. Lo anterior daría pie para suponer la presencia de algún rango de gestión gerencial en pro de una mayor productividad. Sin embargo, la gran mayoría de los resultados, ya anotados, desvirtúan ese supuesto. Cuando se disponga de información adicional sobre la productividad (kilos o toneladas por hectárea y litros de leche por vaca en ordeño), será posible indicar y evaluar con mayor firmeza, la calidad de la gestión gerencial en la zona, uno de cuyos indicadores se refiere a los índices de productividad.

9. Un tema de gran importancia que incluyó esta investigación se refiere al rendimiento o productividad de las áreas sembradas con diferentes cultivos y al número de litros de leche producida por las vacas en ordeño, en las fincas dedicadas a esta actividad.

La productividad señala el grado de eficiencia que presenta una empresa o actividad productiva y depende o se obtiene con el uso económico y racional de los factores de producción. El más importante y valioso de esos recursos es el factor trabajo, representado por los aportes físicos e intelectuales que la fuerza laboral o planta de personal aporta a la empresa en la cual trabaja.

Si el grupo de personas que labora en una unidad de producción tiene suficientes conocimientos de las tareas que debe ejecutar y posee, además, formación, capacitación y experiencia, su aporte o contribución al cumplimiento del objeto social de la empresa será positivo y valioso, porque contribuirá a que sus índices de eficiencia o productividad sean altos.

Por los resultados de las encuestas realizadas en las fincas del Altiplano cundiboyacense se deduce lo siguiente:

1. Predominio de educación y capacitación bajas (58,6\%).

2. Administradores o mayordomos sin ninguna formación en las áreas de la administración (92\%) 
3. Rendimiento de las áreas sembradas y cosechadas inferiores a los promedios de productividad de los mismos cultivos en otras regiones y países (véanse los cuadros Nos.2 y 4 )

De acuerdo con las respuestas a las preguntas de la encuesta, el 55,1\% de los "encargados" o mayordomos de las fincas respondió que no han asistido o participado en cursos de agricultura y ganadería. El 44.8\% contestó afirmativamente.

Un $57.7 \%$ ha asistido a esos cursos en el SENA, el $18.4 \%$ en las UMATAS y el 3.6\% en universidades, lo que indica un vacío grande en el área de la capacitación.

En cuanto se refiere a la preparación en contabilidad, las respuestas del $72.9 \%$ señalan no tenerla y sólo el 27.2\% manifestó que tiene esa preparación y que la utiliza para llevar las cuentas de la finca.

Además, los mayores ingresos anuales procedentes de la explotación de la finca están entre los \$5 y \$50 millones, con porcentajes que fluctúan entre el 22.8\% y el $28.8 \%$.

Un 95.4\% obtiene utilidades que se sitúan entre los \$2 y \$ 10 millones al año. Este resultado conduce a que el $57 \%$ de los propietarios de las fincas consultadas esté satisfecho y un 43\% no lo esté por recibir beneficios que se sitúan entre los $\$ 2$ y $\$ 5$ millones al año.

En cuanto al empleo de crédito, el 49\% lo hace y el $51 \%$ no. Este resultado parece obedecer a la falta de información o desconocimiento de las fuentes y operatividad del crédito que atienden las entidades financieras.

El banco más empleado es el Banco Agrario, que ha sido el agente financiador del 63\% de los encuestados. En $2^{\circ}$ y 3 er. lugar se encuentran el Bancolombia (10.3\% y las Cooperativas (6,3\%) respectivamente.

Por otra parte, el bajo porcentaje ( 14.9\%) de agricultores y ganaderos del Altiplano que pertenece a una asociación, cooperativa o federación, es una confirmación de la idiosincrasia y comportamiento del campesino cundiboyacense, quien sobresale por su individualismo.

Las dos últimas preguntas de la encuesta se refieren a la comercialización de la producción. Las respuestas del 51\% de los entrevistados muestran que venden sus productos utilizando un intermediario, el 20,5\% lo hace directamente a clientela fija, y el $20.4 \%$ comercializa sus productos por medio de acarreo a centros de consumo vecinos. Esas ventas las realizan principalmente en la finca $(43,4 \%)$ y en las plazas de mercado (25\%).

La información anterior permite afirmar que existe una alta dependencia de los intermediarios, lo cual ratificaría la razón por la cual el beneficio neto para el producto rural de esa región (Altiplanicie cundiboyacense) deja mucho qué desear, toda vez que el intermediario recibe una participación mayor al ingreso neto o utilidad que recibe el productor.

Debido a que este documento es un avance preliminar del estudio, no incluye observaciones, resultados y conclusiones más precisos y amplios, en tal forma que apoyen la confirmación de la validez de la hipótesis planteada y los hallazgos o aportes a la disciplina.

\section{Análisis}

Siguiendo el orden de las preguntas contenidas en el cuestionario de la encuesta, su clasificación y tabulación arrojaron los siguientes resultados:

\section{Resultados y efectos de la situación actual del sector}

En la zona seleccionada para esta investigación se encontró que es una realidad el supuesto del cual se parte: una gestión administrativa inexistente o muy ineficiente; personal administrativo con muy baja capacitación; rendimientos por fanegada o por hectárea bajos y, por consiguiente, una productividad baja; rentabilidad de la inversión apenas sufi- 
ciente para la subsistencia de sus propietarios; métodos de explotación o producción obsoletos e inadecuados; ausencia de investigación y de empleo de tecnología avanzada; ausencia de rotación de cultivos y de selección de semillas, y desconocimiento de los métodos modernos de la administración empresarial. Todo lo anterior es una consecuencia del trabajo de campo llevado a cabo en la Altiplanicie cundiboyacense, región seleccionada como muestra representativa o estadística para los departamentos de Boyacá y Cundinamarca.

Una investigación posterior, que puede realizarse en otra zona o región del territorio colombiano, daría a conocer y concluiría que las características de la gestión gerencial en el sector agropecuario tradicional, observadas en la Altiplanicie cundiboyacense se repiten en los demás departamentos colombianos.

\section{ANEXO I}

\section{MUNICIPIOS DE LA ALTIPLANICIE CUNDIBOYACENSE, ESCENARIO PARA EL TRABAJO DE CAMPO}

Facatativá, Zipacón, Mosquera, El Rosal, Madrid, Cajicá, Lenguazaque, Simijaca, Gachetá, Susa, Tabio, Fúquene, Sutamarchán, Cucunubá, Ubaté, Tausa, Sopó, Zipaquirá, Cota, Chía, Carmen de Carupa, Gachancipá, Sutatenza, Tenjo, Nuevo Colón, Boyacá, Funza, Nemocón, Tocancipá, Villapinzón, La Calera, Subachoque, Cogua, Gachalá, Paipa y Villa de Leiva.

Municipios pertenecientes al departamento de Boyacá: veintitrés (23); municipios pertenecientes al departamento de Cundinamarca: trece (13), para un total de treinta y seis municipios seleccionados de la Altiplanicie cundiboyacense.

\section{ANEXO 2}

\section{ENCUESTA UNIVERSIDAD SERGIO ARBOLEDA}

I. Localización de la finca: Municipio

Vereda

2. Extensión de la finca: Fanegadas Hectáreas

3. Es propia Arrendada Contrato de aparcería

4. Actividad principal o única de la finca

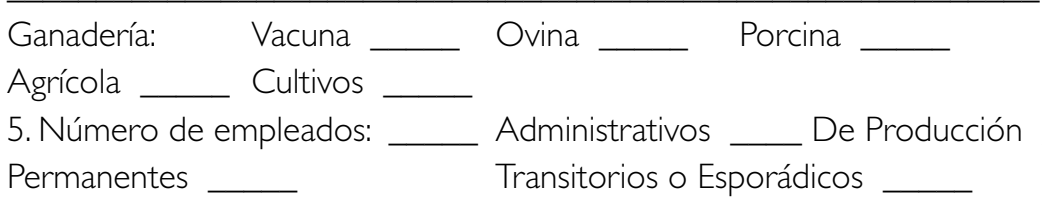

6. Número de Aparcelados o Arrendatarios: Nivel de Escolaridad

7. Miembros de la familia propietaria o administrativa de la finca
8. Emplea maquinaria y equipos
Cuáles
9. Utiliza Agroquímicos:
Cuáles

10. Usted o sus trabajadores han hecho algún curso de agricultura o ganadería

Dónde De cuánta duración

। I. Recibe o ha recibido asistencia o asesoría de alguna entidad

Cuál 
12. La finca lleva contabilidad Sabe cuáles son sus costos de producción

Sabe usted si la finca da utilidades

En cuanto estima estas utilidades al año

$\$ 500.000 \mathrm{a} \mid .000 .000$
de $5.000 .000 \mathrm{a} \mid 0.000 .000 \quad \begin{gathered}1.000 .000 \text { a } 2.000 .000 \\ \text { Más de } \mid 0.000 .000\end{gathered}$ de 2.000 .000 a 5.000 .000

13. Usted y su familia pueden vivir satisfactoriamente con lo que produce la finca

14. Tiene algún crédito

Con quién

15. Está asociado a alguna cooperativa o asociación de productores

Cuál

16. Cuál es la producción por fanegada o hectárea

17. Cómo venden sus productos

En dónde

\section{CUADRO I}

EVOLUCIÓN DEL PIB AGROPECUARIO (Variaciones en porcentajes anuales)

\begin{tabular}{|l|ll|}
\hline $1970-1979$ & $1970-1979$ & $4.48 \%$ \\
\hline $1980-1989$ & $1980-1989$ & $2.94 \%$ \\
\hline $1990-1999$ & $1990-1999$ & $1.72 \%$ \\
\hline $1999-2007$ & $1999-2007$ & $1.91 \%$ \\
\hline
\end{tabular}

Fuente: DANE, DNP y Min-Agricultura (2008)

CUADRO 2

EL SECTOR AGROPECUARIO. RESULTADOS PARA LOS AÑOS I 995 Y 2006

\begin{tabular}{|c|c|c|c|c|c|c|}
\hline CULTIVO & & EAS & & DAS & PRC & CIÓN \\
\hline & 1995 & 2006 & 1995 & 2006 & 1995 & 2006 \\
\hline $\begin{array}{l}\text { Algodón } \\
\text { Arroz }\end{array}$ & & & & & & \\
\hline $\begin{array}{l}\text { Papa } \\
\text { Frijol }\end{array}$ & & & & & & \\
\hline $\begin{array}{l}\text { Maíz } \\
\text { Sorgo } \\
\text { Trigo }\end{array}$ & & & & & & \\
\hline $\begin{array}{l}\text { Banano } \\
\text { Cacao } \\
\text { Caña de Azúcar }\end{array}$ & & & & & & \\
\hline $\begin{array}{l}\text { Café } \\
\text { Palma Africana }\end{array}$ & & & & & & \\
\hline
\end{tabular}




\section{CUADRO 3}

LA POBREZA EN COLOMBIA

(Porcentajes promedio)

\begin{tabular}{|l|l|l|l|}
\hline ANNO & URBANA & RURAL & $\begin{array}{c}\text { INDIGENCIA } \\
\text { (Total) }\end{array}$ \\
\hline & & & \\
\hline & & & \\
\hline & & & \\
\hline
\end{tabular}

Fuente: PNUD, DANE y DNP (2007)

Cuadro elaborado por los autores.

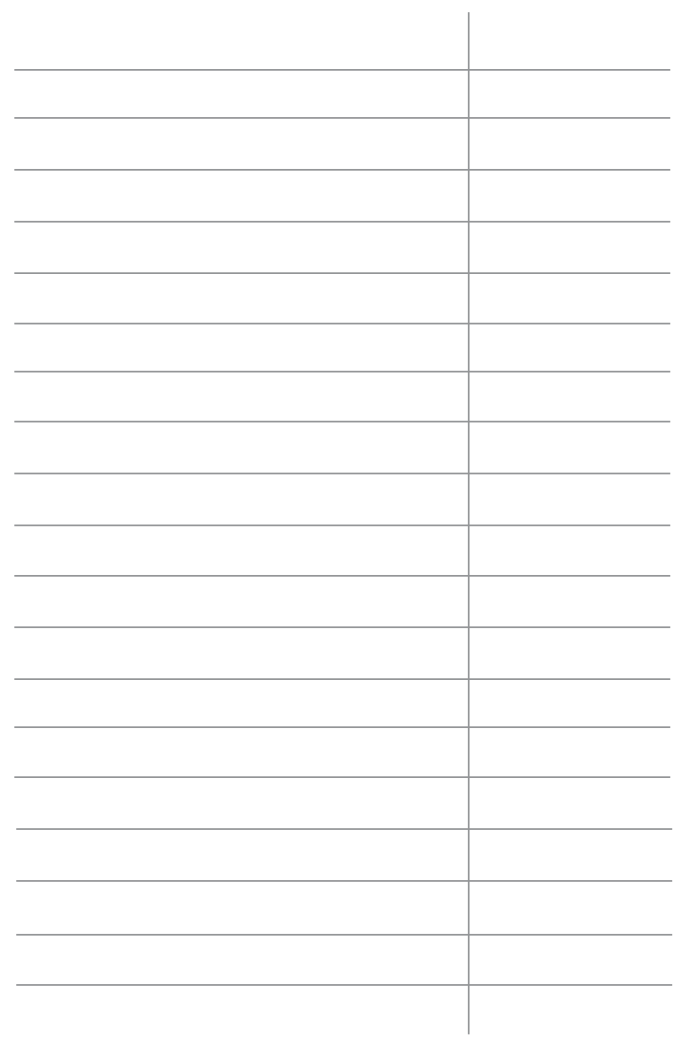




\section{CUADRO 5}

\begin{tabular}{c|c|l}
\multicolumn{3}{c}{ AREA TOTAL CULTIVADA } \\
EN COLOMBIA
\end{tabular}

\begin{tabular}{c|c|l}
\multicolumn{3}{c}{ AREA SEMBRADA CON CULTIVOS } \\
TRANSITORIOS \\
AÑO & HECTÁREAS & \\
\hline 2004 & 1.751 .014 & \\
\hline 2005 & 1.606 .754 & \\
\hline 2006 & 1.550252 & \\
\hline 2007 & 1.545 .900 & \\
\hline 2008 & 2.928 .792 &
\end{tabular}

\section{AREA SEMBRADAS CON CULTIVOS} FORESTALES

\begin{tabular}{c|c|l}
\hline AÑO & HECTÁREAS & \\
\hline 2004 & 204.905 & \\
\hline 2005 & 222.259 & \\
\hline 2006 & 239.067 & \\
\hline 2007 & 270.303 & \\
\hline 2008 & 301.420 &
\end{tabular}

\begin{tabular}{|c|c|}
\hline AÑO & HECTÁREAS \\
\hline 2004 & 2.717 .405 \\
\hline 2005 & 2.780 .267 \\
\hline 2006 & 2.817 .002 \\
\hline 2007 & 2.882 .906 \\
\hline 2008 & 2.928 .792 \\
\hline
\end{tabular}

\begin{tabular}{l|l|l|l} 
& $\begin{array}{c}2006 \\
\text { Jun. - 30 }\end{array}$ & $\begin{array}{c}2007 \\
\text { Jun. - 30 }\end{array}$ & $\begin{array}{c}\text { CRECIMIENTO } \\
\text { Y DISMINUCIÓN }\end{array}$ \\
\hline Total Nal. & & & \\
\hline Agric. - Gan. - Pesca y Caza & & & \\
\hline Ind. Manuf. & & & \\
\hline Comercio - hoteles y & & & \\
restaurantes & & & \\
\hline Servicios (los demás) & & & \\
\hline ZONAS RURALES & & & \\
\hline Agric. - Gan. - Pesca y Caza & & & \\
\hline Ind. Manuf. & & & \\
\hline Comercio - hoteles y & & & \\
\hline restaurantes & & & \\
\hline Servicios (los demás) & & & \\
\hline
\end{tabular}




\section{ANEXO 7}

\section{DISPOSICIONES LEGALES PARA EL SECTOR AGROPECUARIO}

\section{Ley I 133/2007: Agro, Ingreso seguro}

Faculta al Ministerio de Agricultura para invertir en programas para mejorar la productividad del campo y defender los ingresos de los productores agropecuarios. Permite también destinar una línea especial de crédito para reconversión productiva. En el 2008 destinó $\$ 400.000$ millones para financiar 23.000 proyectos de inversión en el sector: \$100.000 millos para proyectos de investigación y desarrollo tecnológico.

\section{Circulares P-49/2007 y P-I I/2008 de la Comisión Nacional de Crédito Agrícola (CNCA):}

Reconocen y otorgan el Incentivo de Capitalización Rural (ICR) a los productores que hagan inversiones nuevas en el sector agropecuario. En 2007, 23.005 productores solicitaron el ICR con créditos por \$ 6 I1.96I millones, de los cuales el 67,5\% fue para pequeños productores.

Res. 26/2008:

Incentiva la asistencia técnica para proyectos productivos agrícolas, pecuarios, avícolas y forestal, con financiación hasta por el $80 \%$ de su valor.

\section{Decreto 2594/2007:}

Reglamenta el Fondo de Inversiones de Capital de Riesgo administrado por FINAGRO, para financiar proyectos agropecuarios, agroindustriales y de reforestación, en zonas con limitaciones para la inversión privada.

\section{Res. 25/Dic./07:}

Fija $\$ 2,75$ billones para créditos a través de FINAGRO. Entre Enero y Mayo de 2008 otorgó 70.448 créditos por $\$ 1,22$ billones a productores agropecuarios.

\section{Ley I I52/07 y Res. No. 7 de Abril/08 de la CNCA:}

Reglamenta una línea especial de crédito para pequeños productores beneficiarios del subsidio para la compra de tierras.

A través del Programa AIS, se crea una línea especial de crédito para incentivar la siembra de alimentos de ciclo corto.

Decretos 4678 y 3363 de 2007:

Establecen las condiciones para la restructuración de cartera adquirida en desarrollo del Programa Nacional de Reactivación Agropecuaria (PRAN).

Res. No. 2, Dic. /07:

La CNCA aprueba el Plan Anual (de Seguros Agropecuarios 2008 y destina $\$ 17.826$ millones para cubrir los riegos de varios cultivos, entre ellos maíz, plátano, arroz y caña de azúcar, en un área total de 123.000 hectáreas.

Res. No. I 16, Marzo/08:

El Ministerio de Agricultura crea el incentivo sanitario para la agricultura de exportación, que incluye a productores de camarón, tilapia y trucha de exportación, con estándares internacionales. Fondos para el incentivo: \$ 10.000 millones.

\section{Programa de Seguridad Alimentaria:}

El Ministerio de Agricultura implementó este Programa para los pequeños agricultores de las zonas paperas de Boyacá, Cundinamarca y Nariño. En el 2008 se des- 
tinaron \$2.160 millones que atenderán a 16.746 familias de 99 municipios con vocación productora de papa.

Sistema de Pago de la Leche cruda al productor:

El Ministerio de Agricultura establece el sistema de pago de la leche cruda para que este sea justo y mantenga la competitividad de la producción nacional.

\section{Fuentes primarias de información}

Para la realización de la investigación, a la cual pertenece el presente avance, se recurrió a fuentes primarias y secundarias. Las primarias corresponden a las personas que respondieron la encuesta en las 386 fincas adicionadas.

Además, se complementaron las informaciones obtenidas en el trabajo de campo con visitas y entrevistas a diferentes funcionarios de las siguientes entidades:

- Ministerio de Agricultura y Desarrollo Rural

- Ministerio de Educación Nacional

- Departamento Administrativo Nacional de Estadística - DANE

- Servicio Nacional de Aprendizaje - SENA

- Instituto Colombiano Agropecuario - ICA

- Corporación de Investigaciones Agropecuarias - CORPOICA

- Federación Nacional de Ganaderos - FEDEGAN

- Sociedad de Agricultores de Colombia - SAC

- Centro de Estudios Ganaderos y Agrícolas - CEGA

- Instituto Geográfico Agustín Codazzi - IGAC

- Banco de la República, Depto. Investigaciones Económicas, Bogotá, D.C.

\section{Bibliografía y Fuentes de Información}

Aguilar Zuluaga, Ignacio, (2008), Principios de Desarrollo Económico; 2a edición, Bogotá: ed. Ecoeditores y Fondo de Publicaciones de la Universidad Sergio Arboleda.

Arango Londoño, Gilberto, (2005), Estructura Económica Colombiana, Bogotá: décima edición; ed. McGraw Hill.

Arias Silva, Andrés, (2008): "El Agro cambia de ritmo en el PIB", Portafolio. Sep. 8, 2008; Bogotá.

Echavarria Olozaga, Hernán (1980), El sentido común de la Reforma Agraria, Bogotá, ed. Andes.

Departamento Nacional de Planeación, (1998) Cambio para Construir la Paz, Bogotá: Tercer Mundo Editores.

Departamento Nacional de Planeación (2005) Visión Colombia II Centenario - 20I9, Bogotá.

Fedegan, (2006), Plan Estratégico de la Ganadería Colombiana, Bogotá: Ed. San Martín Obregón y Cía.

Forero, Jaime, (2002) "Campesinado, economía agraria y sistema alimentario en Colombia"; en Las Dos Colombias: Bogotá: Ed. Norma. 
Instituto Colombiano de Administración (INCOLDA), (1985), La Gerencia en Tiempos Turbulentos, Bogotá: Editorial Andes.

Jhonson, Sherman, (1987), U.S. Department of Agriculture, Washington, D.C.

Kalmanovitz, Salomón (2003), Economía y Nación, Bogotá: Ed. Norma.

Lafaurie, José Félix (2006), Posconflicto y Desarrollo, Bogotá: Ed. Unión Gráfica Ltda.

Lafaurie, José Félix (2007), Pensamiento Económico y Social de Fedegán, Bogotá: Ed. San Martín Obregón \& Cía.

Ministerio de Agricultura y Desarrollo Rural - DNP (2007), Aprovechar las potencialidades del campo, Bogotá.

Ministerio de Agricultura y Desarrollo Rural, (2008); "Perspectivas Agropecuarias - Segundo Semestre 2008", Bogotá.

Roger, Dixey (1976), Agricultural Economy, USA: Ed. lowa State University, University Press, lowa.

Salazar Vargas, Carlos, (2009), Políticas Públicas \& Think Tanks, Bogotá: Ed. Graficolor.

Sudman, Seymour (1976), Applied Sampling (Quantitative studies in social relations), Princeton: Ed. Academic. 